\title{
Prototipo de robot paralelo accionado por cuatro cables: resultados experimentales
}

\section{(Parallel robot prototype driven by four cables: experimental results)}

\author{
Xavier Aguas' ${ }^{1}$, Marco Herrera ${ }^{1}$, Nelson Sotomayor ${ }^{1}$, Oscar Camacho ${ }^{1}$
}

\section{Resumen}

Los robots accionados por cables son una clase especial de robots paralelos formados al reemplazar los enlaces rígidos por cables. Debido a las características que otorgan los cables, como baja inercia y mayor rango de movimiento, pueden realizar una amplia gama de aplicaciones como: la traslación de cámaras de un lugar a otro en eventos deportivos, industria del automovilismo y, principalmente, en la rehabilitación de extremidades. Sin embargo, por la propiedad unilateral de los cables, el mantenerlos tensionados se convierte en un gran desafío. En este artículo se presenta la construcción de un robot paralelo accionado por cuatro cables con el objetivo de trazar tres figuras a través del estudio del modelo cinemático y dinámico que considera la redundancia de actuación, es decir, más cables que grados de libertad, para evitar que requieran mayor tensión durante el movimiento del efector final.

\section{Palabras clave}

cables, cinemática, dinámica, PID, robot.

\begin{abstract}
Parallel cable robots are a special class of parallel robots which are formed by replacing rigid links with cables. Due to the characteristics provided by the cables, like low inertia and greater range of motion, this type of robots can perform a wide range of applications such as: moving cameras from one place to another in sporting events, motoring industry and mainly in rehabilitation of limbs. However, due to the unilateral property of cables, keeping them tensioned becomes a great challenge. This article describes the construction of a parallel robot driven by four cables with the aim to draw three figures through the study of the kinematic and dynamic model considering the redundancy, i.e., more cables than degrees of freedom, in order to avoid those cables that require more tension during the motion of the end-effector.
\end{abstract}

\section{Keywords}

cables, kinematic, dynamic, PID, robot.

\section{Introducción}

En las últimas décadas, los robots accionados por cables han logrado enormes avances en diversos sectores de la industria debido a las ventajas que otorgan respecto de los robots convencionales (manipuladores seriales y paralelos) (Khakpour, Birglen, y Tahan, 2014). Estos robots son utilizados principalmente en aplicaciones de manipulación, soldadura y montaje por ser estructuras simples con un mayor espacio de trabajo y una velocidad de movimiento del efector final más rápida (Pott et al., 2013). Sin embargo, al ser robots accionados por cables presentan nuevos desafíos en el diseño de controladores porque los cables solo pueden ejercer tensión, por esta razón varios investigadores han propuesto adecuar los esquemas de control convencionales utilizados en estructuras seriales y paralelas (Khosravi, Taghirad y Toosi, 2012). En el proceso de diseño de los robots accionados por cables, la simulación del mecanismo es muy 
importante ya que permite tener una nueva idea del movimiento del efector final junto con los cables que lo sujetan y observar cómo el sistema de control desarrollado se adapta al diseño mecánico. Por otro lado, para la implementación de este tipo de robots es esencial considerar el costo de los actuadores porque representan la principal contribución en el costo total de la estructura. Por lo tanto, al ser una estructura simple que usa un número reducido de componentes conduce a un diseño rentable por la transportabilidad, facilidad de montaje y desmontaje, reconfigurabilidad y mantenimiento (Anson, 2015). Los robots accionados por cuatro cables con dos grados de libertad, poseen dos movimientos traslacionales, en el eje $x$ y en el eje $y$, donde cuatro cables son sujetados en un punto específico conocido como efector final (Williams II y Gallina, 2003). Las principales características que poseen son su baja inercia, su baja fricción y su gran destreza de movimiento en el espacio de trabajo. Actualmente, estos robots se encuentran en aplicaciones para limpieza de vidrios, arado de tierra en pequeños terrenos, dispositivos médicos para rehabilitación (Zanotto, Rosati, Minto y Rossi, 2014) y como dispositivos hápticos, los cuales están atrayendo el interés de muchos investigadores (Gallina, Rosati, y Rossi, 2001). La presente investigación es una extensión del trabajo presentado en INCISCOS denominado "A Sliding Mode Control for a Planar 4-Cable Direct Driven Robot" (Aguas, Herrera, Camacho y Leica, 2018), en el cual se describe el estudio realizado para el diseño y la construcción de un prototipo de robot paralelo accionado por cuatro cables con dos grados de libertad en el plano $x y$. Las pruebas desarrolladas fueron realizadas a través del software computacional MATLAB, donde se analiza la prueba para determinar la repetitibilidad del robot para el trazado de figuras bajo un patrón de referencia.

\section{Metodología}

En esta sección se desarrolla el análisis del modelo cinemático y dinámico del robot accionado por cuatro cables de dos grados de libertad con el fin de diseñar el controlador tipo PID para trazar cuatro figuras diferentes las cuales se muestran en la Tabla 1. Además, se diseña la estructura del prototipo, a través de un software de diseño asistido por computadora (CAD) denominado Autodesk Inventor Professional 2016, a partir del espacio de trabajo y del uso de servomotores de la marca Dynamixel AX-12A. Asimismo, se desarrolla el diseño electrónico para controlar el prototipo mediante la memoria compartida, utilizando la Librería SAS Tools para el software MATLAB para la comunicación entre la computadora y el prototipo. Finalmente, se realizan pruebas para determinar la repetibilidad del prototipo y se muestra los trazos realizados sobre los patrones de referencia establecidos. La metodología aplicada en esta investigación se divide en cuatro etapas:

- Análisis del modelo cinemático y dinámico

- Diseño mecánico y electrónico

- Diseño del controlador tipo PID

- Diseño del software de control

Estas etapas son muy importantes para el funcionamiento del prototipo y se detallan a continuación:

Los robots accionados por cuatro cables con dos grados de libertad proporcionan dos movimientos traslacionales tanto en el eje $x$ como en el eje $y$, donde cuatro cables son sujetados en un punto denominado como efector final. Las características que posee este robot son 
baja inercia, baja fricción y gran destreza de movimiento en el espacio de trabajo. El diseño del prototipo propuesto es considerado con redundancia de actuación, es decir, más cables que grados de libertad para evitar que algunos requieran mayor tensión o que el efector pueda perder el control en algún punto del espacio de trabajo cuando uno de los cables no mantenga la tensión necesaria (Khosravi y Taghirad, 2013).

\section{Modelo cinemático de posición del robot paralelo accionado por cuatro cables}

El modelo cinemático de este robot relaciona las longitudes de los cables con la posición del efector final, como también el cálculo inverso desde la posición del efector final a las longitudes de los cables. Las relaciones mencionadas se conocen como cinemática directa e inversa, respectivamente. Para realizar el análisis del modelo cinemático del robot paralelo accionado por cuatro cables se considera que todos los cables siempre se mantienen en tensión. La Figura 1 describe el esquema del modelo cinemático, donde el origen de la referencia está dado por el vector $\mathbf{X}_{\mathbf{0}}=\left[\begin{array}{ll}x_{0} & y_{0}\end{array}\right]^{T}$ el cual se encuentra ubicado en el centroide de la base cuadrada de lado $L_{B^{*}}$ Cada uno de los actuadores se encuentra en la posición dada por $\mathbf{A}_{\mathbf{i}}=\left[A_{i x} A_{i y}\right]$; la longitud de los cables es descrito por $L_{i^{\prime}}$ los ángulos por $\theta_{i}$, donde $i=1,2, \ldots, 4$ y la posición del efector final del robot es dado por el vector $\mathbf{X}_{\mathbf{e}}=\left[x_{e} y_{e}\right]^{T}$.

Figura 1. Esquema para el estudio del modelo cinemático del robot paralelo accionado por cuatro cables

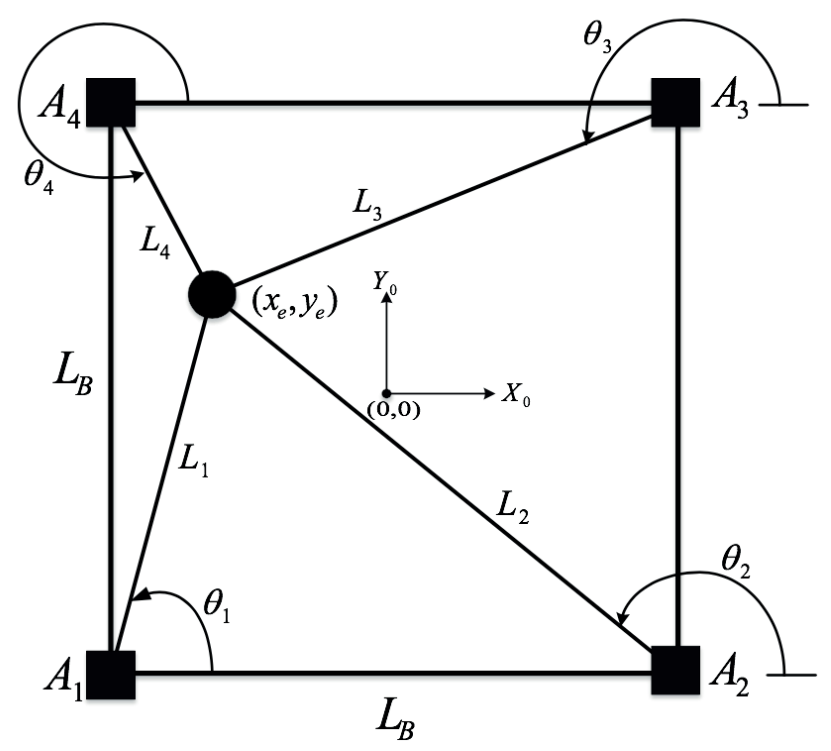

Solamente calculando la norma entre la posición del efector final y las posiciones de los actuadores $A_{i^{\prime}}$ la cinemática inversa es obtenida (Aguas, Cuaycal, Paredes y Herrera, 2018). En la Ecuación 1 se describe como obtener la longitud de los cables $L_{i}$ a partir de la posición del efector final $\mathbf{x}_{\mathbf{e}}=\left[x_{e} y_{e}\right]^{T}$.

$$
L_{i}=\sqrt{\left(x_{e}-A_{i x}\right)^{2}+\left(y_{e}-A_{i y}\right)^{2}}
$$


La solución de la posición del efector final mostrada en la Ecuación 2 está dada al realizar la intersección de dos circunferencias, la primera centrada en $A_{1}$ con radio $L_{i}$ y la otra centrada en $A_{2}$ con radio $L_{2} 1$

$$
x_{e}=\frac{L_{B}^{2}+L_{1}^{2}-L_{2}^{2}}{2 L_{B}} y_{e}= \pm \sqrt{L_{1}^{2}-x_{e}^{2}}
$$

Para afirmar que la posición del efector final se encuentre dentro de la base cuadrada se elige la solución positiva y posteriormente se realiza el cambio de referencia al centroide del cuadrado. Este método es aplicado para cualquier robot planar accionado por cables.

\section{Modelo dinámico del robot paralelo accionado por cuatro cables}

El modelo dinámico relaciona el movimiento del efector final con los torques requeridos por los actuadores. Este modelo es requerido cuando existen altas velocidades y aceleraciones en el movimiento del efector final (Gallina, Rossi y Williams II, 2001). El modelo resultante del robot paralelo accionado por cuatro cables es acoplado y altamente no lineal (Aguas, Herrera, Camacho y Leica, 2018). El modelo del efector final, del sistema de enrollamiento y del sistema en conjunto se presentan a continuación:

\section{Modelo dinámico del efector final}

Para definir el modelo dinámico del efector final se requiere el uso de la Segunda Ley de Newton, la cual establece que la aceleración de un objeto es directamente proporcional a la fuerza total que actúa sobre él e inversamente proporcional a su masa. La Ecuación 3 expresa la ley mencionada:

$$
\mathbf{m} \ddot{\mathbf{X}}_{\mathbf{e}}=\mathbf{F}_{\mathbf{R}}
$$

Donde:

$\mathbf{m}=\left[\begin{array}{cc}m & 0 \\ 0 & m\end{array}\right]$ : Matriz de masas,

$\mathbf{X}_{\mathbf{e}}=\left[x_{e} y_{e}\right]^{T}$ : Posición del efector final,

$\mathbf{F}_{\mathbf{R}}=\left[f_{x} f_{y}\right]^{T}$ : Fuerza resultante de todos los cables (tensiones) actuando sobre el efector final.

\section{Modelo dinámico del sistema de enrollamiento}

Para definir el modelo dinámico del sistema de enrollamiento se considera el comportamiento dinámico del eje unido con la polea que enrolla el cable. El diagrama de cuerpo libre para el eje del motor junto con la polea para el $i$-ésimo cable se muestra en la Figura 2. En este diagrama, el ángulo de la polea $\beta_{i}=0$ cuando el efector final se encuentra localizado en el centroide del cuadrado, desde esta posición es medido este ángulo, en el caso del $\theta_{i}$ debe ser de acuerdo a la posición de cada actuador. 
Figura 2. Sistema eje del motor-polea

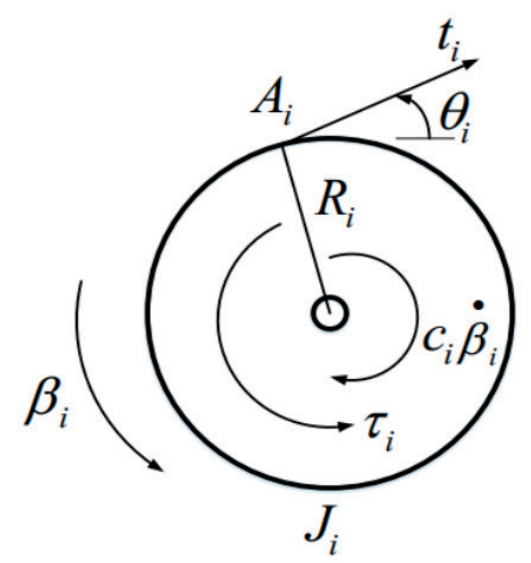

La Ecuación 4 se determina a partir del diagrama de cuerpo libre mostrado anteriormente donde se observa cómo se combina el eje del motor y la polea de enrollamiento:

$$
\mathbf{J} \ddot{\boldsymbol{\beta}}+\mathbf{C} \dot{\boldsymbol{\beta}}=\boldsymbol{\tau}-R \mathbf{T}
$$

Donde:

$\mathbf{J}=\left[\begin{array}{ccc}J_{1} & 3 & 0 \\ \hdashline & \ddots & \vdots \\ 0 & & J_{4}\end{array}\right]:$ Matriz diagonal de inercias rotacionales de los motores,

$\mathbf{C}=\left[\begin{array}{ccc}c_{1} & 3 & 0 \\ \hdashline & \ddots & \vdots \\ 0 & \vdots & c_{4}\end{array}\right]$ Matriz diagonal de coeficientes de viscosidad de los motores,

$\boldsymbol{\beta}$ : Vector de ángulos de las poleas,

T. Vector de tensiones,

$\boldsymbol{\tau}$ : Vector de torques generado por los motores,

$R$ : Radio de la polea.

Con el fin de presentar las tensiones que ejercen los cables en función de la posición angular y los torques de los motores, se obtiene lo siguiente:

$$
\mathbf{T}=\frac{1}{R}(\boldsymbol{\tau}-\mathbf{J} \ddot{\boldsymbol{\beta}}-\mathbf{C} \boldsymbol{\beta})
$$

\section{Modelo dinámico del sistema}

El modelo dinámico del sistema se encuentra combinando las ecuaciones dinámicas de movimiento del efector final y del sistema de enrollamiento, junto con la cinemática inversa de posición que relaciona los ángulos de las poleas $\beta_{i}(i=1, \ldots, 4)$ a partir de la posición del efector final $\mathbf{x}_{\mathbf{e}}=\left[x_{e} y_{e}\right]^{T}$. El modelo general del sistema puede ser expresado como la ecuación de la robótica, a través de la relación estática $S T=F_{R}$ mostrada en (Aguas et al., 2018) y es representado por la Ecuación 6: 


$$
\left[R \mathbf{m}+\mathbf{S J}\left(\frac{\partial \boldsymbol{\beta}}{\partial \mathbf{X}_{\mathbf{e}}}\right)\right] \ddot{\mathbf{X}}_{\mathbf{e}}+\mathbf{S}\left[\mathbf{J}\left(\frac{\mathrm{d}}{\mathrm{dt}}\left(\frac{\partial \boldsymbol{\beta}}{\partial \mathbf{X}_{\mathbf{e}}}\right)\right)+\mathbf{C} \frac{\partial \boldsymbol{\beta}}{\partial \mathbf{X}_{\mathbf{e}}}\right] \dot{\mathbf{X}}_{\mathbf{e}}=\mathbf{S} \boldsymbol{\tau}
$$

Escribiendo la Ecuación 6 de forma simplificada, se obtiene:

$$
\mathbf{M}\left(\mathbf{X}_{\mathrm{e}}\right) \ddot{\mathbf{X}}_{\mathrm{e}}+\mathbf{N}\left(\mathbf{X}_{\mathrm{e}}, \dot{\mathbf{X}}_{\mathrm{e}}\right) \dot{\mathbf{X}}_{\mathbf{e}}=\mathbf{S \tau}
$$

Donde:

$$
\begin{aligned}
& \mathbf{M}\left(\mathbf{X}_{\mathbf{e}}\right)=R \mathbf{m}+\mathbf{S} \mathbf{J} \frac{\partial \boldsymbol{\beta}}{\partial \mathbf{X}_{\mathbf{e}}}: \text { Matriz de inercia, } \\
& \mathbf{N}\left(\mathbf{X}_{\mathbf{e}}, \dot{\mathbf{X}}_{\mathbf{e}}\right)=\mathbf{S}\left[\mathbf{J} \frac{\mathrm{d}}{\mathrm{dt}}\left(\frac{\partial \boldsymbol{\beta}}{\partial \mathbf{X}_{\mathbf{e}}}\right)+\mathbf{C} \frac{\partial \boldsymbol{\beta}}{\partial \mathbf{X}_{\mathbf{e}}}\right]: \text { Matriz de Coriolis, } \\
& \text { S: Matriz Jacobiano estático, }
\end{aligned}
$$

\section{Diseño mecánico}

El diseño de las partes mecánicas es realizado en un software de diseño asistido por computadora (Software CAD) denominado Autodesk Inventor Professional 2016 que ofrece herramientas versátiles para diseño mecánico y validación de las piezas antes de fabricarlas.

Para el diseño de las poleas se consideró un radio de $0.03 \mathrm{~m}$ para que en cada vuelta la polea enrolle o desenrolle $0.18 \mathrm{~m}$ de cable. La Ecuación 8 muestra el cálculo de la longitud del cable que abarca en una vuelta de la polea.

$$
L_{\text {cable }}=2 \pi R=2 \pi(0.03)=0.1884 \mathrm{~m} / \text { vuelta }
$$

En el diseño de las poleas el parámetro más relevante es el diámetro del hilo $(1 \mathrm{~mm})$ porque es de vital importancia que pueda alojarse sin salirse de las ranuras durante el giro de las poleas. En el diseño del roscado, el parámetro conocido como paso fue escogido en un rango de 3 a $5 \mathrm{~mm}$, el cual define la distancia que existe entre dos hilos adyacentes. Por otro lado, con el estudio previo realizado en la selección de los actuadores, los servomotores Dynamixel AX-12A al ser configurados en modo 'articulación' (modo para recibir valores de ángulos) tienen el giro restringido hasta $300^{\circ}$; por esta razón, se debe implementar un sistema de engranajes que permitan a las poleas girar por lo menos tres veces más de lo que el servomotor brinda para recoger o desplegar cable para realizar las figuras propuestas. A través de la herramienta para generar engranajes que posee este software CAD permite el diseño rápido y eficiente de engranajes, se diseña el sistema de transmisión a través de engranajes para aumentar el giro de los motores y por ende el giro de las poleas. El módulo de los engranes diseñados es 0.83, este parámetro identifica a un conjunto de engranajes permitiendo conocer las dimensiones tanto de los dientes como de todo el engranaje, en otras palabras dos engranajes que se acoplen correctamente tienen el mismo módulo Además, se diseña los soportes para los actuadores de acuerdo a las dimensiones de los servomotores Dynamixel AX-12A y a la separación que debe existir entre los engranajes para que se acoplen correctamente. Esta pieza cuenta con perforaciones para que los tornillos pasen a través de él y se sujete a la base cuadrada del prototipo de robot paralelo accionado por cuatro cables. Finalmente, la base del robot es una placa cuadrada de Madera MDF de $0.7 \mathrm{~m}$ de lado y un grosor de $0.006 \mathrm{~m}$ para que no exista deformaciones por el peso del efector final y se coloca un vidrio que representa el área de trabajo del robot con el 
objetivo de reducir la fricción del efector final durante su movimiento. La base cuenta con perforaciones para sujetar los actuadores y los soportes del prototipo. La Figura 3 ilustra el prototipo de un robot paralelo accionado por cuatro cables.

Figura 3. Prototipo de un robot paralelo accionado por cuatro cables
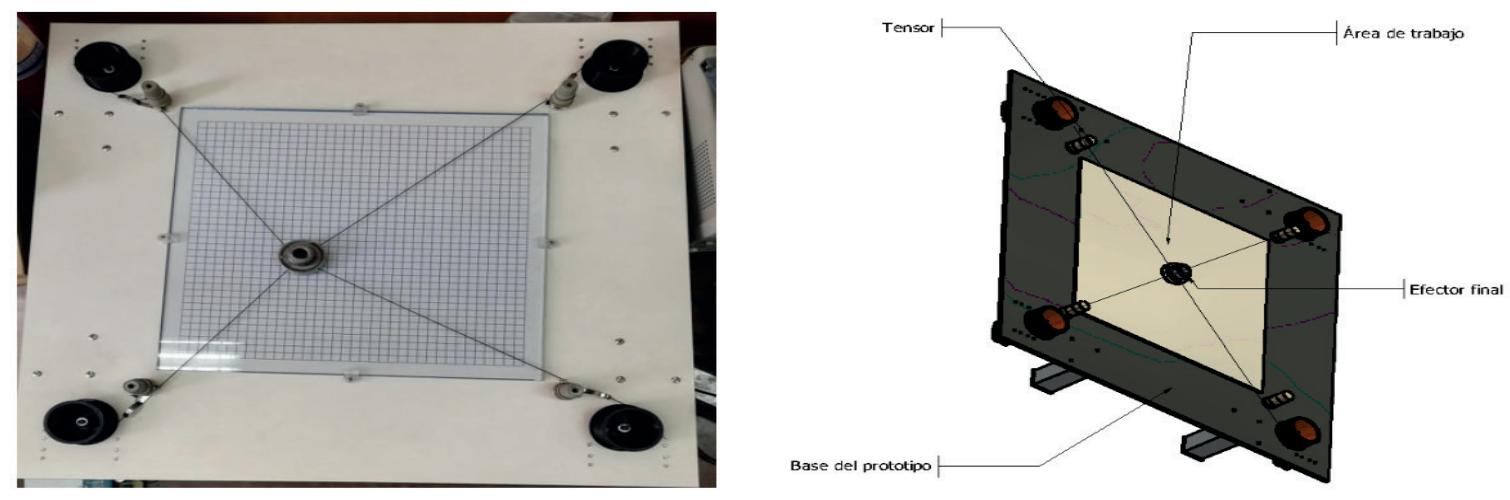

\section{Diseño electrónico}

Para realizar el diseño la placa de control integrando la tarjeta de control ARDUINO y el circuito integrado 74HC241/126 para el control de los servomotores del prototipo se utiliza el software Proteus Design Suite 8.6, el cual permite la construcción de placas electrónicas a partir del diseño y simulación. Activando uno de los puertos seriales (UART o USART) de la tarjeta de control ARDUINO se puede comunicar con el servomotor Dynamixel AX-12A. El diseño de la placa de control final del prototipo es realizado con un estilo actual conocido como SHIELD de tal manera que los circuitos modulares se monten para ampliar las capacidades de la tarjeta ARDUINO. Generalmente en el diseño se considera la forma estándar del ARDUINO con el espacio de los pines para que pueda encajarse sin problemas. La placa consta de puertos para conectar los visualizadores leds y botones para que el robot se posicione en una de las esquinas del espacio de trabajo para borrar la figura trazada y otro botón para que regrese a su posición de origen (centroide de base cuadrada).

\section{Diseño del software de control}

El desarrollo del software de control está compuesto por tres partes. La primera se encarga de obtener la posición de los servomotores a través de la lectura de los encoders con el sistema embebido ARDUINO. La segunda parte es una aplicación desarrollada en C++ a través del software VISUAL STUDIO 2012 que se encarga de comunicar la computadora con la tarjeta embebida ARDUINO para enviar y recibir los datos de ángulo correspondientes de cada uno de los actuadores. Por otro lado, la última etapa procesa toda la información adquirida y comanda el robot por medio de una memoria compartida de la librería SAS TOOLS en el software MATLAB 2017A para realizar el trazado de figuras predefinadas. El esquema del software de control del robot paralelo accionado por cuatro cables es representado en la Figura 4. 
Figura 4. Esquema del software de control del robot paralelo accionado por cuatro cables

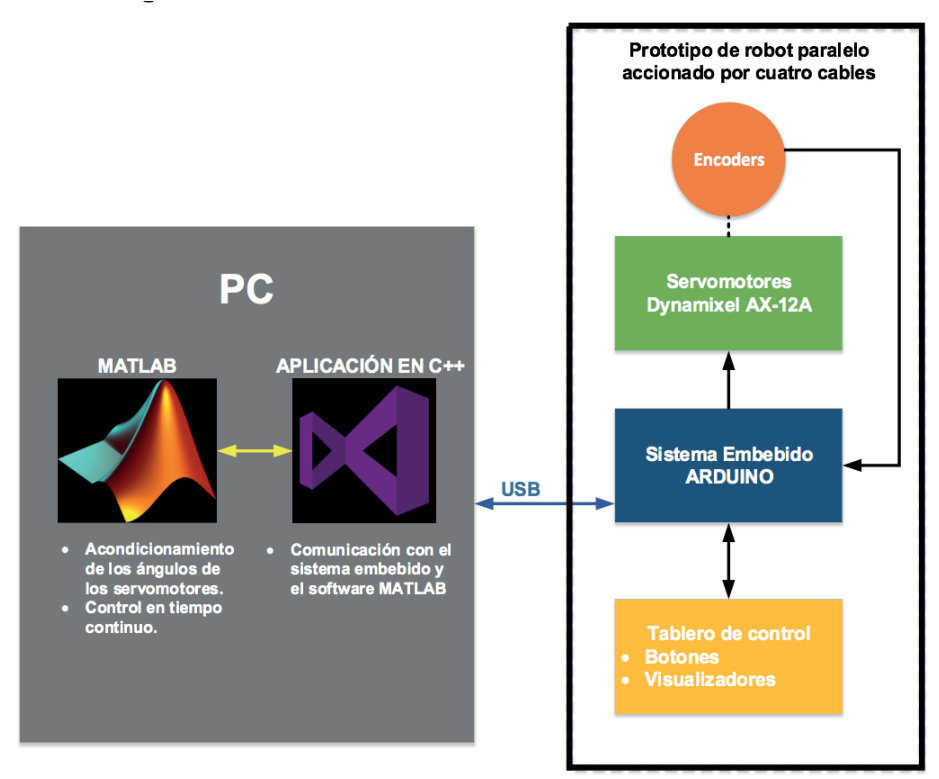

La trama de datos se basa en una comunicación maestro-esclavo, donde la computadora realiza la petición de los datos de posición de cada servomotor de forma periódica al sistema embebido ARDUINO para ser procesadas y visualizadas en la interfaz gráfica. La trama de datos del esclavo contiene un encabezado y los datos correspondientes de posiciones de los servomotores leídos a través de los encoders, por otro lado, la trama de datos del maestro contiene el encabezado y los ángulos deseados que deben posicionarse los servomotores. Es importante recalcar que los datos que se envía o recibe son de 16 bits los cuales son separados en dos datos de 8 bits para formar la trama a una velocidad de 115200 bps. Por otro lado, a través de la librería SAS TOOLs se puede emplear el bloque de memoria compartida en Simulink, el cual se caracteriza porque puede configurarse de acuerdo a los requerimientos de la aplicación a desarrollarse, es decir, el usuario puede elegir el número de entradas y salidas que requiera. La memoria compartida para el prototipo de robot paralelo accionado por cuatro cables cuenta con cuatro entradas y salidas respectivamente, cada uno de los datos recibidos y enviados son de tipo flotante. Las entradas son las posiciones deseadas de cada servomotor y las salidas son las posiciones actuales de los mismos.

Figura 5. Bloque de memoria compartida del prototipo para MATLAB/Simulink

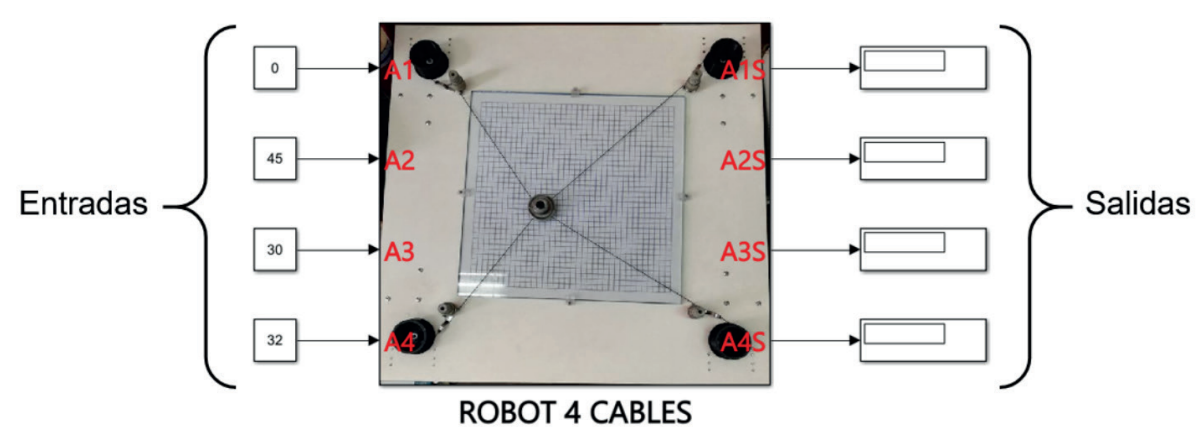




\section{Desarrollo del controlador para el robot paralelo accionado por cuatro cables}

Para garantizar que cada componente del vector $\boldsymbol{\tau}$ sea positivo o al menos cero se introduce una fuerza virtual $F_{v}$.

$$
\mathbf{F}_{\mathbf{v}}=\mathbf{S \tau}
$$

Con esta consideración se desarrolla una ley de control con el fin de que todos los componentes del vector de torque $\boldsymbol{\tau}$ sean positivos cuando la posición del efector final está dentro del espacio de trabajo estático. La ley de control propuesta en este estudio es un control PI para asegurar que el efector final llegue al punto deseado. Para el diseño del controlador, el error de seguimiento es definido como:

$$
\mathbf{e}(t)=\mathbf{X}_{\mathbf{d}}(\mathbf{t})-\mathbf{X}_{\mathbf{r}}(\mathbf{t})
$$

Donde:

$X_{d}(t)$ : Vector de posición deseado.

$X_{r}(t)$ : Vector de posición del efector final del prototipo.

El controlador PI tiene la siguiente forma:

$$
\operatorname{PI}(t)=F_{v}(t)=K_{p} e(t)+K_{i} \int e(t) d t
$$

Los valores de los parámetros de sintonización del controlador para el trazado de figuras son $K_{p}=\left[\begin{array}{ll}10 & 10\end{array}\right]$ y $K_{i}=[0.010 .01]$.

El esquema de control mostrado en la Figura 6 funciona de la siguiente manera: el controlador PI genera una fuerza virtual $\mathbf{F}_{\mathbf{v}}(\mathbf{t})=\left[f_{x} f_{y}\right]^{T}$ y, a través del cálculo de la relación de fuerza a torques (Ecuación 9), permite que las acciones de control cambien garantizando que la tensión de los cables se mantenga durante el movimiento del efector final. Estas acciones de control $\boldsymbol{\tau}(\mathbf{t})$ ingresan al modelo del robot paralelo accionado por cuatro cables con el fin de obtener los valores de ángulos deseados de las poleas $\boldsymbol{\beta}_{\mathbf{d}}(\mathbf{t})$ que serán enviadas al prototipo desarrollado. Cada actuador tiene un controlador tipo PID, el cual ha sido configurado a través del propio software que brinda el fabricante para garantizar que el servomotor llegue a la posición angular deseada. Es primordial mencionar que no se puede obtener directamente la posición del efector final del prototipo $\mathbf{X}_{\mathbf{r}}(\mathbf{t})=\left[x_{r} y_{r}\right]^{T}$, mediante los encoders de cada actuador, por esta razón se utiliza una codificación con la lectura de los encoders de cada ángulo de las poleas $\boldsymbol{\beta}(\mathbf{t})$ para determinar las longitud de los cables $L(t)$ a través de la Ecuación 12.

$$
\mathbf{L}(\mathbf{t})=\left[\begin{array}{c}
L_{01}-r \beta_{1} \\
\vdots \\
L_{04}-r \beta_{4}
\end{array}\right]
$$

Donde:

$$
\begin{aligned}
& \boldsymbol{L}_{\mathbf{0 1}}, \ldots, \boldsymbol{L}_{\mathbf{0 4}} \text { : Longitud inicial de los cables, } \\
& \boldsymbol{\beta}_{\mathbf{1}}, \ldots, \boldsymbol{\beta}_{\mathbf{4}} \text { : Ángulos de los servomotores. }
\end{aligned}
$$


Una vez obtenidas las longitudes de los cables es necesario utilizar la cinemática directa de posición presentada en la Ecuación 2 que relaciona la longitud de los cables y la posición del efector final con el fin de realimentar dicha posición.

Figura 6. Esquema de control implementado para el robot paralelo accionado por cuatro cables en MATLAB/Simulink

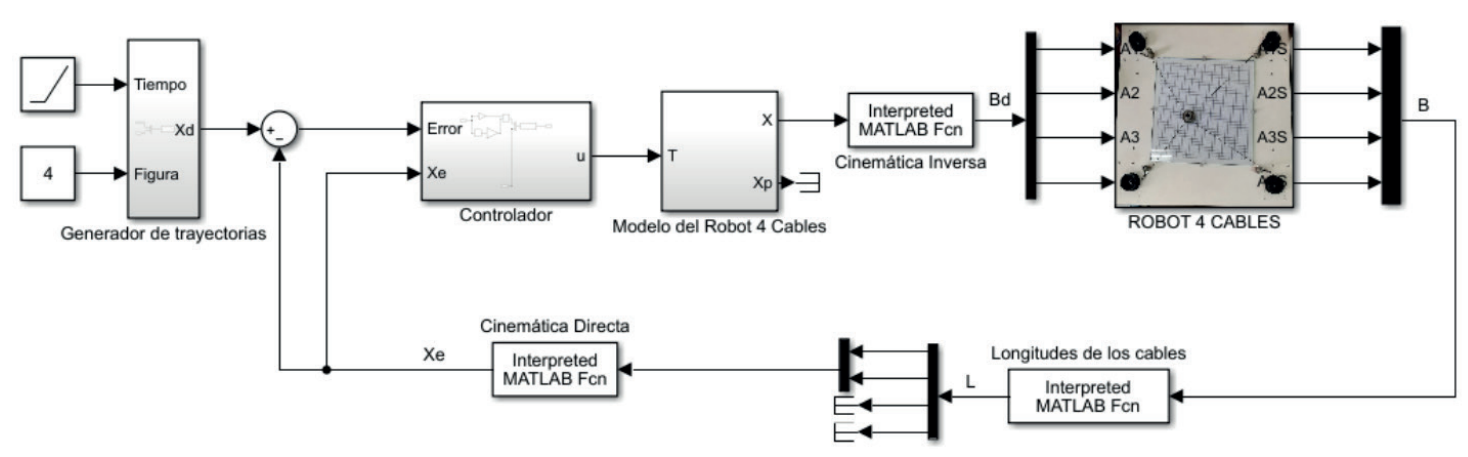

El esquema de control propuesto, únicamente trabajará correctamente si se mantiene una tensión suficiente en todos los cables durante el movimiento del efector final.

\section{Resultados}

Las pruebas realizadas fueron ejecutadas en una computadora Intel(R)Core(TM) i7-5500U CPU a 2.40GHz con 8GB de memoria RAM con un tiempo de muestreo de 0.1 y usa el método ODE45 (Solve non-stiff differential equations). El objetivo es seguir una trayectoria para el trazado de una figura propuesta con el efector final del robot paralelo accionado por cuatro cables. Para determinar la repetibilidad del robot desarrollado y mostrar que el error no procede del controlador diseñado, sino que es propio del prototipo, se realizó esta prueba por medio del trazado del círculo propuesto en la Tabla 1. La repetibilidad es definida como la capacidad que posee un robot de pasar por un punto determinado las veces que sea. Este parámetro muestra el grado de exactitud que existe en la repetición de las trayectorias que se realiza para el trazado de las figuras (Heredia \& Mena, 2017). La Ecuación 13 representa la desviación estándar, la cual muestra la dispersión de los datos en una prueba.

$$
\delta=\sqrt{\frac{\sum_{i=1}^{N}\left(x_{i}-\bar{x}\right)^{2}}{N}}
$$

Donde:

$\delta$ : Desviación estándar,

$X_{i}$ : Valor de un conjunto de datos,

$\bar{x}$ : Media de un conjunto de datos,

$N$ : Número de trayectorias 
Con el fin de obtener la desviación estándar de la trayectoria de la figura realizada se debe encontrar un promedio de los valores calculados para en cada instante de tiempo en el cual se ha muestreado la posición a través de la Ecuación 14.

$$
\bar{\delta}=\sqrt{\frac{\sum_{i=1}^{M} \delta_{i}}{M}}
$$

Donde:

$\bar{\delta}$ : Desviación estándar promedio,

$\delta_{i}$ : Valor de la desviación estándar en cada instante de tiempo (0.1 segundos),

M: Número de datos.

Para hallar la repetibilidad del robot paralelo accionado por cuatro cables, se realizan cinco círculos de $9 \mathrm{~cm}$ de radio. El círculo de referencia es representado con una línea sólida de color azul, mientras que los círculos que traza el robot son ilustrados con diferentes colores; además, las pruebas de repetibilidad del prototipo se realizan para el eje $x$ como para el eje $y$, lo cual determinará la repetibilidad del robot. La trayectoria trazada en el los ejes coordenados es mostrado en la Figura 8:

Figura 8. Prueba de repetibilidad del prototipo para el circulo propuesto
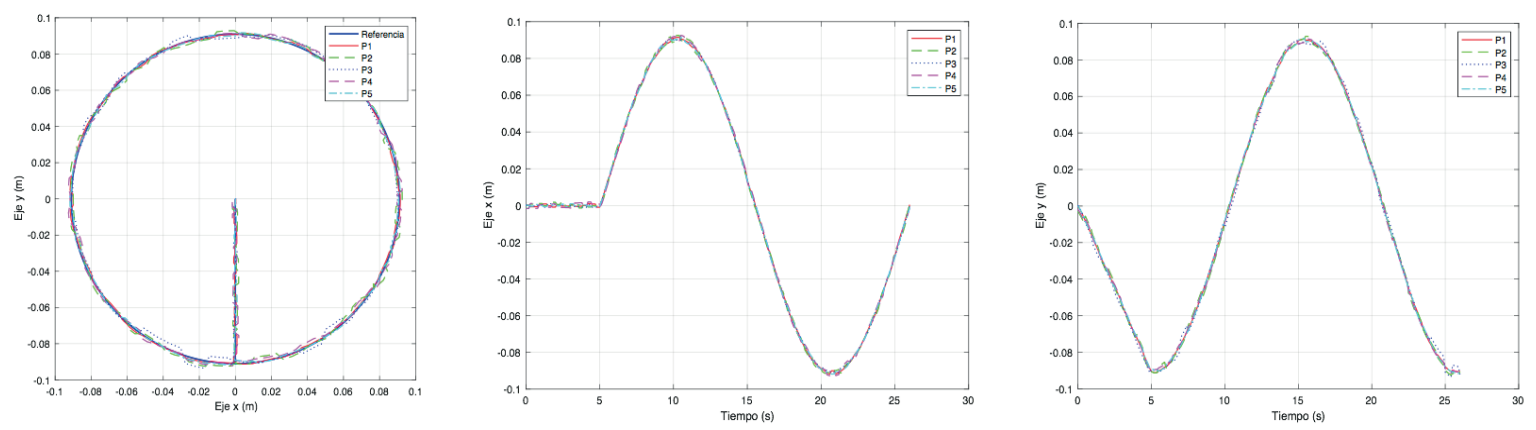

El valor de la desviación estándar es calculado por medio de la Ecuación 13 en cada instante de tiempo en cada eje coordernado y la Ecuación 14 presenta la desviación estándar promedio para el eje $x$ como para el eje $y$ con un valor de $0.3 \mathrm{~mm}$ y $0.7 \mathrm{~mm}$, respectivamente. La repetibilidad es considerada seis veces el valor de la desviación estándar (Heredia y Mena, 2017), es decir, $2.2 \mathrm{~mm}$ para el eje $x$ y $4.2 \mathrm{~mm}$ para el eje $y$. Estos valores de repetibilidad garantizan un $99.8 \%$ de probabilidad de que el prototipo traze las figuras por el rango de valores determinado y muestran el error propio de la planta independiente de la actuación del controlador diseñado. Realizada la prueba de repetibilidad, el prototipo se encuentra listo para realizar cualquier tipo de tarea implementada en el software MATLAB/SIMUINK, en este caso, el objetivo es seguir una trayectoria para el trazado de cuatro figuras con el efector final del robot paralelo accionado por cuatro cables. Las figuras desarrolladas son descritas en la Tabla 1 gracias a sus ecuaciones paramétricas: 
Tabla 1. Figuras propuestas para el trazado del robot paralelo accionado por cuatro cables

\begin{tabular}{|c|c|c|c|c|}
\hline Círculo & & Cuadrado & & Flor de cuatro pétalos \\
\hline $\begin{array}{l}x(t)=\left\{\begin{array}{cc}0 & 0 \leq t \leq 5 \\
0.09 \cdot \cos \left(0.2 t-\frac{\pi}{2}\right) & 5<t \leq 35\end{array}\right. \\
y(t)=\left\{\begin{array}{cc}\frac{-0.1 \cdot t}{5} & 0 \leq t \leq 5 \\
0.09 \cdot \sin \left(0.2 t-\frac{\pi}{2}\right) & 5<t \leq 35\end{array}\right.\end{array}$ & $x(t)=$ & $\begin{array}{l}\left\{\begin{array}{c}\frac{0.15 \cdot t}{8} \\
0.15-\frac{0.3(t-8)}{8} \\
-0.15 \\
-0.15+\frac{0.3(t-24)}{8} \\
0.15\end{array}\right. \\
\left\{\begin{array}{c}\frac{0.15 \cdot t}{8} \\
0.15-\frac{0.3(t-16)}{8} \\
-\frac{0.15}{-0.15+\frac{0.3(t-32)}{8}}\end{array}\right.\end{array}$ & $\begin{array}{c}0 \leq t \leq 8 \\
8<t \leq 16 \\
16<t \leq 24 \\
24<t \leq 32 \\
32<t \leq 40 \\
0 \leq t \leq 8 \\
8<t \leq 16 \\
16<t \leq 24 \\
24<t \leq 32 \\
32<t \leq 40\end{array}$ & $\begin{array}{c}x(t)=0.13 \cdot \sin (2 t) \cos (t) \\
y(t)=0.13 \cdot \sin (2 t) \sin (t)\end{array}$ \\
\hline
\end{tabular}

Para mostrar el funcionamiento del prototipo diseñado se coloca un marcador de color azul en el efector final del robot para que traze las figuras propuestas mencionadas anteriormente. La Figura 9 muestra el patrón de referencia de color rojo junto con la figura elaborada por el robot para observar la similitud entre ellas.

Figura 9. Figuras realizadas por el prototipo siguiendo un patrón de referencia establecido

\begin{tabular}{|c|c|c|c|}
\hline Círculo & Cuadrado \\
\hline & & \\
\hline
\end{tabular}

Para realizar el análisis de las figuras trazadas por el robot se utilizó un software de diseño gráfico para colocar el patrón de referencia establecido para cada caso. Al momento de colocar la referencia se observó que los trazos del robot tienen una leve inclinación de aproximadamente $1.27^{\circ}$ en sentido antihorario. Sin embargo, cada figura presenta una gran similitud con su respectiva referencia, con un error en los extremos de las figuras debido a la perdida momentánea de tensión por un sútil juego mecánico (movimiento involuntario) entre el acople de los dos engranes diseñados para aumentar el giro de las poleas. En fin, el sistema propuesto únicamente trabajará correctamente si solo se mantiene una tensión suficiente en todos los cables durante el movimiento del efector final.

\section{Conclusiones y recomendaciones}

Se desarrolló un robot paralelo accionado por cuatro cables para realizar el trazado de tres figuras utilizando del modelo cinemático y dinámico. La dimensión del robot fue determinada a partir del espacio de trabajo que va a ser requerida para llevar a cabo el trazado de las figuras propuestas (Cuadrado de $40 \mathrm{~cm}$ de lado). El controlador propuesto otorgó una repetibilidad de 
$2.2 \mathrm{~mm}$ para el eje $x$ y $4.2 \mathrm{~mm}$ para el eje $y$. Asimismo, al colocar el patrón de referencia sobre la figura trazada por el prototipo se evidenció una inclinación de $1.27^{\circ}$ en sentido antihorario, esta inclinación fue notoria en la figura del cuadrado.

\section{Referencias}

Aguas, X.; Herrera, M.; Camacho, O., y Leica, P. (2018). A Sliding Mode Control for a Planar 4-Cable Direct Driven Robot. En: 2018 International Conference on Information Systems and Computer Science (INCISCOS) (pp. 23-28). https://doi.org/10.1109/INCISCOS.2018.00011

Aguas, X. I.; Cuaycal, A.; Paredes, I., y Herrera, M. (2018). A Fuzzy Sliding Mode Controller for Planar 4-Cable Direct Driven Robot. Enfoque UTE, 9(4), 99-109.

Anson, M. (2015). Cable-driven parallel manipulators with base mobility: A planar case study (PhD Thesis). State University of New York at Buffalo.

Gallina, P.; Rosati, G., y Rossi, A. (2001). 3-d.o.f. Wire Driven Planar Haptic Interface. Journal of Intelligent and Robotic Systems, 32(1), 23-36. https://doi.org/10.1023/A:1012095609866

Gallina, P.; Rossi, A., y Williams II, R. L. (2001). Planar cable-direct-driven robots, part ii: Dynamics and control. En ASME. DECT2001 ASME Design Engineering Technical Conference. Pittsburgh: ASME Publisher (Vol. 2, pp. 1241-1247).

Heredia, J., y Mena, S. (2017). Implementación de un manipulador móvil para desarrollar tareas de seguimiento de trayectoria con un controlador tipo PID. Escuela Politécnica Nacional, Quito. Recuperado de http://bibdigital.epn.edu.ec/handle/15000/17238

Khakpour, H.; Birglen, L., y Tahan, S. A. (2014). Synthesis of Differentially Driven Planar Cable Parallel Manipulators. IEEE Transactions on Robotics, 30(3), 619.630. https://doi.org/10.1109/ TR0.2013.2295891

Khosravi, M. A., y Taghirad, H. D. (2012). Dynamic Analysis and Control of Cable Driven Robots with Elastic Cables. Transactions of the Canadian Society for Mechanical Engineering, 35(4), 543-557.

Khosravi, M., y Taghirad, H. (2013). Experimental Performance of Robust PID Controller on a Planar Cable Robot. En T. Bruckmann \& A. Pott (eds.), Cable-Driven Parallel Robots (pp. 337-352). Berlin, Heidelberg: Springer Berlin Heidelberg. https://doi.org/10.1007/978-3-642-31988-4_21

Pott, A.; Mütherich, H.; Kraus, W.; Schmidt, V.; Miermeister, P.; Dietz, T., \& Verl, A. (2013). Cable-driven parallel robots for industrial applications: The IPAnema system family. En IEEE ISR 2013 (pp. 1-6). https://doi.org/10.1109/ISR.2013.6695742

Williams II, R., y Gallina, P. (2003). Translational Planar Cable-Direct-Driven Robots. Journal of Intelligent and Robotic Systems, 37(1), 69-96. https://doi.org/10.1023/A:1023975507009

Zanotto, D.; Rosati, G.; Minto, S., y Rossi, A. (2014). Sophia-3: A Semiadaptive Cable-Driven Rehabilitation Device With a Tilting Working Plane. IEEE Transactions on Robotics, 30(4), 974979. https://doi.org/10.1109/TR0.2014.2301532 